\title{
Evaluation of bipolar lengths of the kidneys in healthy Sri Lankan adults in view of assessing the need for nomograms
}

\author{
Kodikara $\mathbf{I}^{1}$, Gamage $\mathbf{D}^{2}$, Nanayakkara $\mathbf{G}^{1}$, Ilayperuma $\mathbf{I}^{1}$ \\ ${ }^{I}$ Department of Anatomy, Faculty of Medicine, University of Ruhuna, Galle, Sri Lanka. \\ ${ }^{2}$ Base Hospital Tissamaharama, Sri Lanka.
}

Correspondence: Dr. Iroshani Kodikara

e-mail: iroshanikodikara@gmail.com

(7) https://orcid.org/0000-0001-8534-4571

\begin{abstract}
Introduction: Renal sizes are of immense use to diagnose renal pathologies. As ethnic variations are known to influence renal sizes, we aimed to assess the ethnic variations in bipolar renal length (RL) and kidney to body height ratio (KBR) of a young adult Sri Lankan population.

Methods: Healthy adults were recruited, by excluding subjects with previous renal surgeries, renal diseases, or other chronic illnesses. Bilateral RLs were measured sonographically.

Results: The study sample consisted of 215 individuals (100 females) with an age range of 19-36 years (mean age $=24$ years). Mean right RL was $9.77 \pm 0.78 \mathrm{~cm}$; mean left RL was $10.16 \pm 0.83 \mathrm{~cm}$; mean KBR of the right kidney was $0.60 \pm 0.05 \mathrm{~mm} / \mathrm{cm}$ and left kidney was $0.63 \pm 0.05 \mathrm{~mm} / \mathrm{cm}$. Right and left RLs and KBRs of males were $9.82 \pm 0.76 \mathrm{~cm} ; 10.3 \pm 0.78 \mathrm{~cm}$ and $0.59 \pm 0.05 \mathrm{~mm} / \mathrm{cm} ; 0.62 \pm 0.05 \mathrm{~mm} / \mathrm{cm}$ respectively and same parameters in females were $9.71 \pm 0.8 \mathrm{~cm} ; 10.0 \pm 0.9 \mathrm{~cm}$ and $0.62 \pm 0.05 \mathrm{~mm} / \mathrm{cm} ; 0.64 \pm 0.05 \mathrm{~mm} / \mathrm{cm}$ respectively. Irrespective of gender, left RL and KBR were higher than those of the right side ( $\mathrm{p}<0.001)$. RLs were significantly different $(\mathrm{p}<0.05)$ compared to European and North American populations.
\end{abstract}

Conclusions: This preliminary study can be considered as the first step in establishing age and gender specific reference values for RL and KBR for a Sri Lankan population. Due to ethnic variations, accuracy of using renal nomograms from other populations as reference values raises serious concerns.

Keywords: Bipolarlength, healthy adults, kidney to body height ratio, ultrasonography

\section{Introduction}

Kidneys are paired retroperitoneal organs serving an excretory function. Renal size alterations are frequently described in various renal pathologies, while the renal size is a decisive factor in obtaining a successful renal biopsy sample (1). For many more reasons, including diagnosis and management of renal pathologies, having an accurate understanding of normative renal sizes is essential. While bipolar renal length (RL) has been considered the traditional measurement in renal size assessment, kidney to body height ratio (KBR) has recently described as a more reliable parameter due to minimal gender and body height dependency $(2,3)$.

Organ morphometry can be assessed during cadaveric / surgical dissections or using radiological images. Although cadaveric specimens resemble in vivo human organs with fair accuracy, a certain degree of tissue alterations during the preservation process could influence the organ size $(4,5)$. Whereas, ultrasonography is of immense use in assessing organ morphometry due to its real-time imaging facility, non-invasiveness, free availability, safety and cost-effectiveness while having proven reliability in renal size measurements $(1-3,6)$. 
Renal size is dependent on several factors such as age, gender, and height of the subject. Previously described ethnic variations in renal sizes are perhaps partially representative of variations in age, gender, or height of the subject $(3,7,8)$. Since data regarding normal renal dimensions are scarce for the Sri Lankan population, normal reference values are obtained from Western populations. In Western countries, bipolar length below $9 \mathrm{~cm}$ is considered as the threshold to diagnose irreversible renal failure; however, this value appears to be within normal range for some Asian populations (8). Thus, the use of Western reference ranges may lead to a falsepositive diagnosis of renal diseases (8). Hence, it is exigent to have benchmark renal parameters for the Sri Lankan population. This need is further underscored by the possibility for diagnostic and therapeutic decisions to be influenced by falsepositive renal measurements. Therefore, in this study, we evaluated the normal RL and KBR of a selected healthy young adult Sri Lankan population to establish population-specific reference values and to describe geographical variations in such parameters.

\section{Methods}

A retrospective, cross-sectional review of patients, presenting to a health care centre in the Southern Province of Sri Lanka for medical checkups from March 2015 to March 2017, identified as the $(n=215)$ study subjects. Ethical approval for the study was obtained from the Ethical Review Committee of Kotealawala Defense University of Sri Lanka (RP/2018/13). Age and sex-matched male and female study subjects were within 18 - 40 years of age. The subjects were excluded from the study if they were found to have serum creatinine values $>1.5 \mathrm{mg} / \mathrm{dL}$ (done within last three months), diagnosis of chronic illness, acute illness at the time of investigation, past history of renal calcular disease or renal surgeries, recurrent urinary tract infections, history of childhood renal diseases; renal cysts, asymptomatic renal calculi, unilateral kidney or other congenital morphological renal anomalies, or discrepancy of $>1.5 \mathrm{~cm}$ between right and left bipolar lengths. The eligible subjects were identified by analysing the clinical history through review of the available medical records.
Subjects' date of birth was used to calculate the age, and the personal height was measured using a stadiometer, in standing erect position without footwear, according to the standard protocols.

Ultrasonographic renal evaluation was performed by an experienced radiologist using the $3.5 \mathrm{MHz}$ curvilinear probe of the high-end ultrasound scanner GE Volusion scanner (Seongnam, Gyeonggi, Korea; released to the market in 2016). All of them were fully hydrated and had a full bladder at the time of the investigation. The subjects were lying on left lateral recombinant position for the right kidney examination and vice versa for the left kidney examination. All the measurements were obtained during full inspiration while taking precautions to avoid freezing an oblique image of the kidney. Maximum pole to pole length of each kidney was measured in the frozen image to the nearest $0.1 \mathrm{~mm}$ (Figure 1). Each measurement was repeated thrice, and the average was taken for calculations.

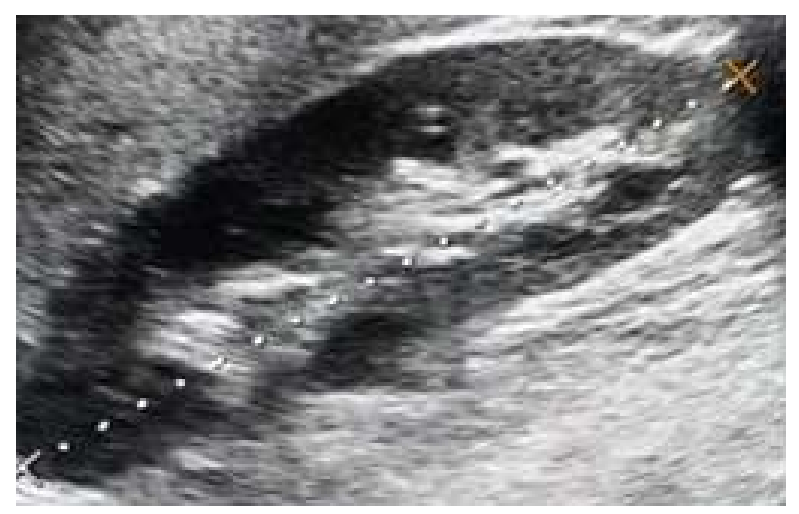

Figure 1: Ultrasound image of kidney showing bipolar renal length measurement (white dotted linebipolar length)

The kidney to body height ratio (KBR) was calculated for each kidney by the following: dividing the renal length in millimeters by the subject's body height expressed in centimeters (2).

\section{Statistical analysis}

Statistical analysis was performed using SPSS 20 IBM statistical software. Categorical variables were expressed as percentages, and continuous variables were expressed as means, standard deviations, ranges, percentiles and inter-quartile ranges. 
The right and left renal lengths were compared using paired sample T-test, and gender variation of the measurements were compared using an independent sample T-test. $\mathrm{P}$ values less than 0.05 were considered as statistically significant.

\section{Results}

Out of all studied subjects $(n=215), 46.5 \%(n=100)$ were females and $53.4 \%(n=115)$ were males; no significant age difference was observed among male and female study populations $(\mathrm{T}=0.376 ; \mathrm{p}>0.05)$. Mean age and height (2 SD) of the study population was $24(4.3)$ years and $162.0(8.15) \mathrm{cm}$, respectively. The baseline characteristics of the study sample and the renal measurements are tabulated (Table 1). The left kidney was longer than the right kidney $(p<0.001)$ with a mean difference of $0.39 \mathrm{~mm}$ between right and left kidneys. Left KBR was higher than right $\operatorname{KBR}(\mathrm{p}<0.001)$ with a mean difference of $0.03 \mathrm{~mm} / \mathrm{cm}$.
Basic demographic data and renal sizes of male and female study populations are tabulated (Table 2). Male subjects were taller than female subjects $(T=11.914 ; \mathrm{p}<0.001)$. Similarly, the males had longer kidneys compared to females $(\mathrm{p}=0.01)$, while mean renal length difference of the right and the left kidney of male and female study population was $0.11 \mathrm{~cm}$ and $0.25 \mathrm{~cm}$, respectively. By contrast, females had higher KBR values compared to males $(p<0.001)$. Though a significant gender influence was shown on right $\mathrm{KBR}(\mathrm{T}=4.938$; $\mathrm{p}<0.0001)$ and left KBR $(\mathrm{T}=3.107 ; \mathrm{p}<0.002)$, the gender influence was shown only on the left renal length $(\mathrm{T}=-2.56 ; \mathrm{p}=0.011$; versus right renal length: $\mathrm{T}=1.04 ; \mathrm{p}=0.298$ ).

Table 1: Baseline characteristics and renal measurements of the study sample

\begin{tabular}{lllc}
\hline Parameters & & $\begin{array}{c}\text { Right kidney } \\
(\mathrm{n}=215)\end{array}$ & $\begin{array}{c}\text { Left kidney } \\
(\mathrm{n}=215)\end{array}$ \\
\hline RL $(\mathrm{cm})$ & Mean (SD) & $9.77(0.78)$ & $10.16(0.83)^{* *}$ \\
& Range & $8.1-12.34$ & $7.8-12.36$ \\
& Median & 9.8 & 10.2 \\
& Inter quartile range & $9.2-10.2$ & $9.6-10.7$ \\
& $10^{\text {th }}-90^{\text {th }}$ percentiles & $8.8-10.8$ & $9.0-11.4$ \\
$\mathrm{KBR}(\mathrm{mm} / \mathrm{cm})$ & Mean $(\mathrm{SD})$ & $0.60(0.05)$ & $0.63(0.05)^{* *}$ \\
& Range & $0.47-0.75$ & $0.46-0.78$ \\
& Median & 0.60 & 0.63 \\
& Inter quartile range & $0.57-0.63$ & $0.59-0.66$ \\
& $10^{\text {th }}-90^{\text {th }}$ percentiles & $0.54-0.67$ & $0.56-0.70$ \\
\hline
\end{tabular}

RL- renal length; KBR- kidney to body height ratio; ** $\mathrm{p}<0.001$

Table 2: Baseline characteristics \& renal measurements of male and female study populations

\begin{tabular}{|c|c|c|}
\hline & Male $(n=115)$ & Female $(n=100)$ \\
\hline Age (years) & $24 \quad(4.5)$ & $24 \quad(4.06)$ \\
\hline Height (cm) & $166.79(6.3)$ & $156.49(6.3)$ \\
\hline $\mathrm{R} / \mathrm{K}$ length $(\mathrm{cm})$ & $9.82(0.76)^{*}$ & $9.71(0.8)^{*}$ \\
\hline L/K length $(\mathrm{cm})$ & $10.30(0.78)^{*}$ & $10.0(0.9)^{*}$ \\
\hline $\mathrm{R} / \mathrm{K} \mathrm{KBR}(\mathrm{mm} / \mathrm{cm})$ & $0.59(0.05)^{* *}$ & $0.62(0.05)^{* *}$ \\
\hline $\mathrm{L} / \mathrm{K} \mathrm{KBR}(\mathrm{mm} / \mathrm{cm})$ & $0.62(0.05)^{* *}$ & $0.64(0.05)^{* *}$ \\
\hline
\end{tabular}

$\mathrm{R} / \mathrm{K}$ - right kidney, L/K- left kidney, KBR- kidney to body height ratio; results are expressed as mean (SD); * $\mathrm{p}<0.05$; ** $\mathrm{p}<0.001$ 


\section{Discussion}

While renal sizes are a surrogate indicator of renal function, they often are the cornerstones of diagnosing and monitoring renal pathologies. Among numerous non-invasive technologies that assess renal sizes, ultrasonography is widely used due to its free availability and cost effectiveness. Whilst the renal length (RL) or the bipolar length has been the most frequently measured parameter, there has been increasing interest in measuring kidney to body height ratio (KBR) for renal size assessment (2). Here we report normal values for both RL and KBR for young adult Sri Lankan population; both RL and KBR of the left side were found to be higher than those of the right side. We also report a gender variation in both RL and KBR with males having longer kidneys whilst the opposite was true for KBR.

In comparison to the RL that were reported for a Sri Lankan farming population from North Central Province, this study reports slightly longer right and left kidneys for both genders. However, the RL difference between two populations was not statistically significant $(\mathrm{T}=1.73 ; \mathrm{p}=0.11$; Figure 2$)$ (9).
Reasons for this discrepancy in RL among Sri Lankan populations could be multi-factorial. First, Nadeeshani, et al. recruited subjects with a wide age range ( $<40$ years to $<60$ years). Perhaps their mean RL would have been influenced by the age-dependent gradual decrease in renal mass, which is particularly obvious in the subjects older than 60 years $(3,8)$. Secondly, the farmers from North Central Province might be suffering from subclinical chronic kidney disease of which the renal size reduction could be considered as an early feature (10).

The mean renal lengths of the studied Sri Lankan population were compared with renal lengths of other populations using independent sample T-test. Renal lengths of the Sri Lankans were compatible with those reported for Indian $(\mathrm{T}=1.53 ; \mathrm{p}=0.13)$ and Malaysian $(\mathrm{T}=0.41 ; \mathrm{p}=0.36)$ populations $(7,8)$. By contrast, it was significantly smaller than Kuwaiti $(\mathrm{T}=3.73 ; \mathrm{p}=0.03)$, Mexican $(\mathrm{T}=2.58 ; \mathrm{p}=0.06)$, Croatian $(\mathrm{T}=0.539 ; \mathrm{p}=0.014)$ and Iranian $(\mathrm{T}=4.72$; $\mathrm{p}=0.02)$ populations $(3,11-, 13)($ Fig. 2$)$.

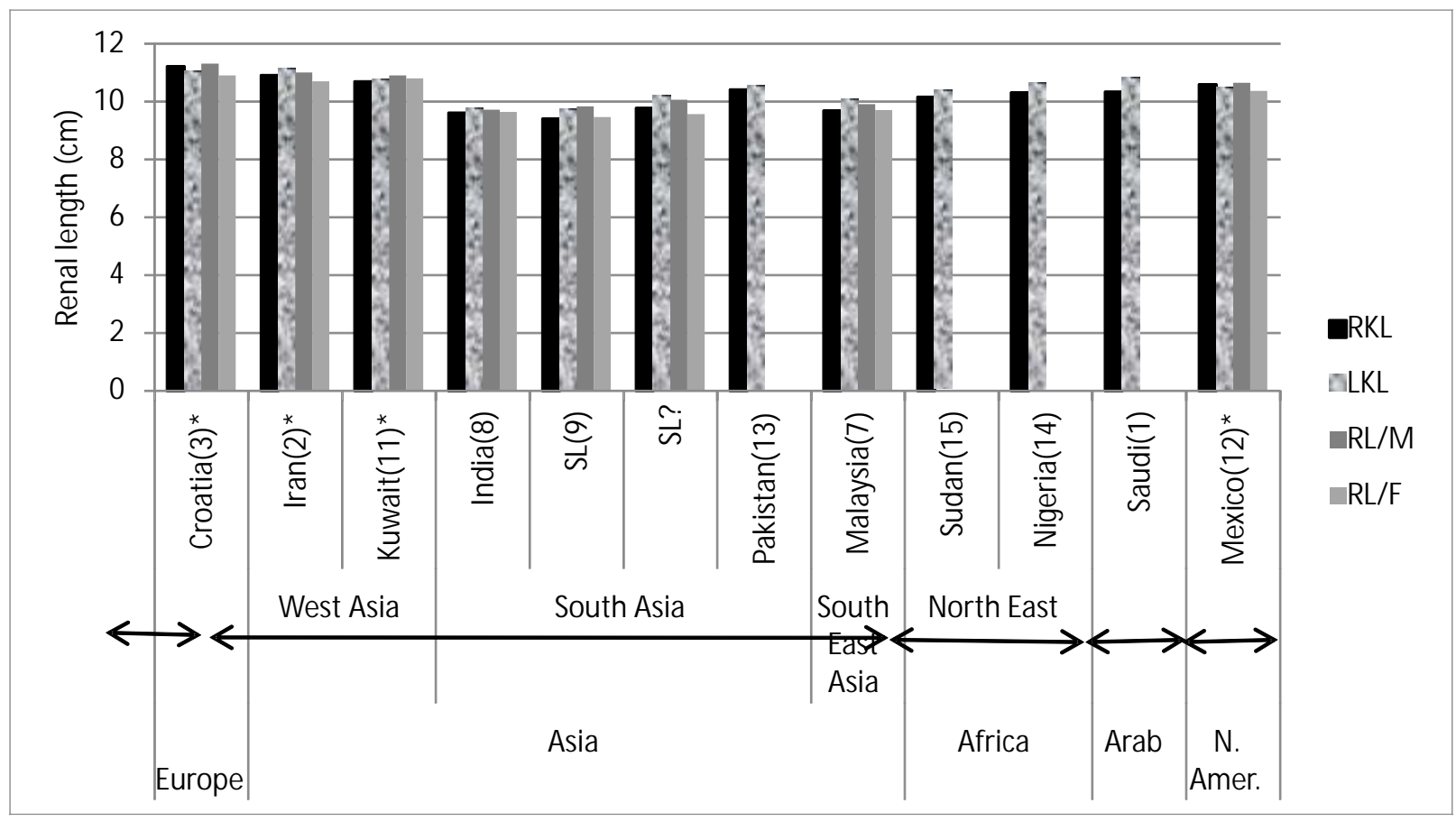

(RKL- right kidney length; LKL- left kidney length; RL/M- renal length in males; RL/F- renal length in females; SL?- current study; SL- Sri Lanka; N. Amer.- North America; *p<0.05); (1-3,7-9,11-15)

Figure 2: Comparison of bipolar lengths among different population 
Except for Sudanese population, our cohort had significantly smaller kidneys compared to Pakistani $(\mathrm{T}=2.98 ; \mathrm{p}=0.05)$, Saudi $(\mathrm{T}=1.95 ; \mathrm{p}=0.09)$, Nigerian $(\mathrm{T}=1.97 ; \mathrm{p}=0.09)$ and Sudanese $(\mathrm{T}=1.28 ; \mathrm{p}=0.16)$ populations $(1,13-15)$. Collectively a clear national variation in the renal lengths is demonstrated. Moreover, perhaps a geographical influence is contributing to the observed ethnic variation in renal lengths in the following way: renal lengths of populations from Europe, Western Asia, and North America were significantly longer than those from South-Asian populations, whilst renal lengths of populations from Africa and the Middle-East were not significantly different from those reported for South-Asian and South-East-Asian populations (Figure 2) $(1,3,7,8,11,12,15)$. Since the renal sizes of the Pakistani population has shown a minor deviation from the rest of South-Asian populations, factors such as genetic influence and nutritional pattern on the body composition might also influence on renal sizes (13).
Therefore, ethnic as well geographical influences on renal length need to be considered carefully; hence considering normative parameters from remote geographical regions as the reference values need to be reconsidered. Further, having an insight into renal sizes of different ethnicities would help develop medical technologies such as and angiogram catheters.

Though the difference was not significant, the KBR values of the Croatian $(T=1.99 ; \mathrm{p}=0.09)$ and Iranian $(\mathrm{T}=2.59 ; \mathrm{p}=0.02)$ populations were found to be higher than the Sri Lankan values (Figure 3) $(2,3)$. Taking into account that these populations have demonstrated a significant ethnic variation in renal length; KBR would likely serve as a better parameter due to minimum ethnic influence. However, considering the scarcity of KBR values specific to different populations, evidence is still insufficient to postulate conclusions on applications of KBR.

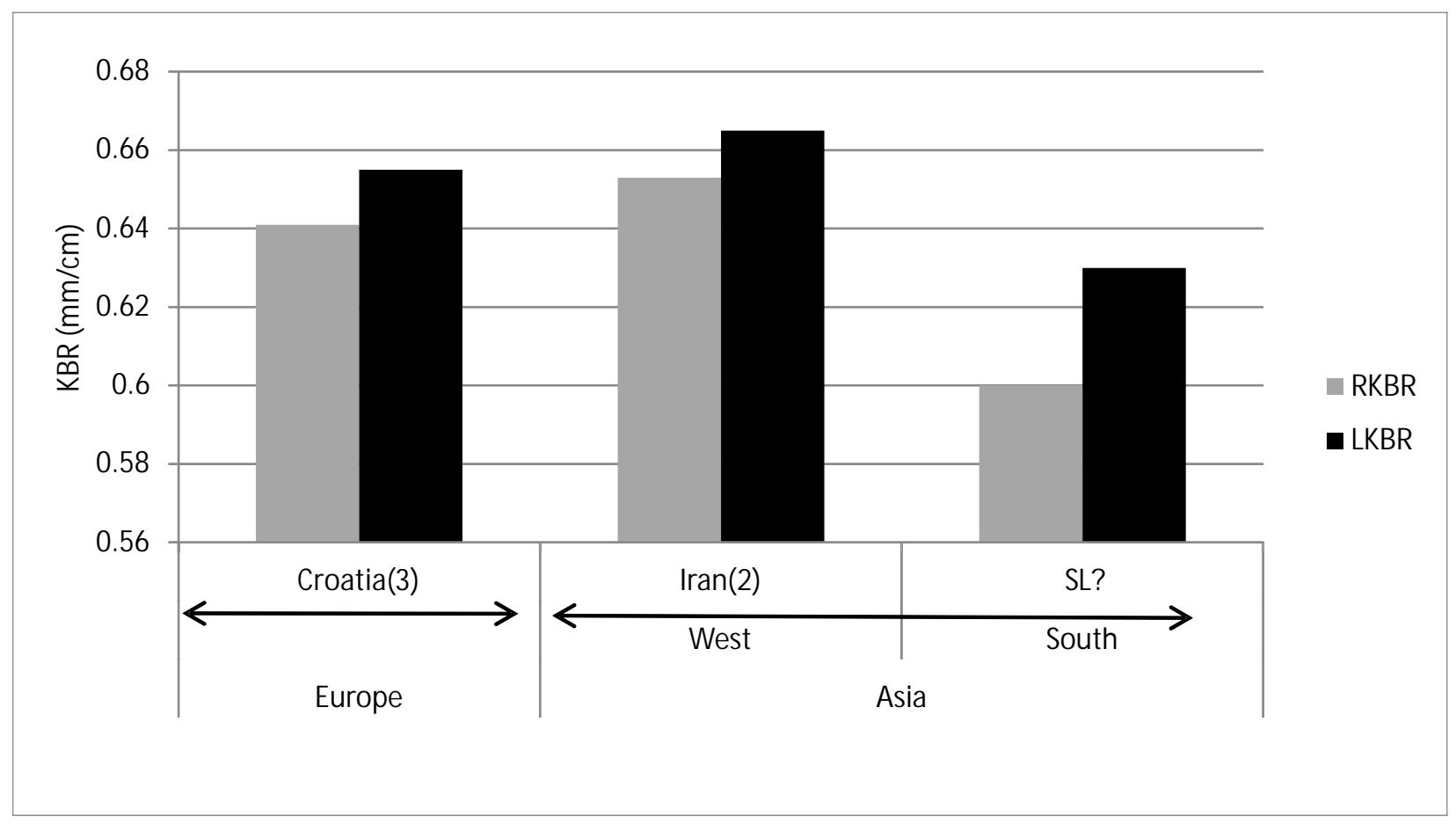

(RKBR- right kidney to body height ratio; LKBR- left kidney to body height ratio; KBR- kidney to body height ratio; SL? current study) $(3,2)$

Figure 3: Comparison of KBR among different populations 
Though the left kidney is reported to be significantly longer than the right kidney $(1,7,8,11,15,16)$, few studies have reported a reciprocal finding $(3,12)$. This study is in agreement with several other studies that reported a significant KBR difference between right and left kidneys $(2,3)$. Similarly, the left kidney reported to have a higher volume than the right kidney. It is known that the renal volume is influenced by the tissue mass in the kidney (16). Thus, there appears to be a discrepancy in renal tissue mass and the number of nephrons in either side.

The current study has shown a significant gender difference only for the left RL, while some other studies have reported a gender difference for both kidneys (2,3). Similarly, we have shown a significant gender difference for bilateral KBRs, contradicting the findings of several other studies those have reported lack of gender variability in $\operatorname{KBR}(2,3)$. Whether such difference in RL and KBR among populations has arisen due to ethnic influences or merely due to statistical artefacts needs to be considered in large population based studies.

A strength of this study is that bipolar lengths were assessed by a single experienced radiologist, thus inter-observer variability has been eliminated. Further, since the low inter and intra-observer variability in sonographic assessment of renal lengths has been demonstrated in multiple studies, the use of ultrasonography is fully justified to assess renal lengths (17). This study is limited by a relatively small sample size of a single geographical region, and it should be considered before generalization of study findings to the larger Sri Lankan population. Assessment of other linear or volumetric renal parameters along with RL and KBR would further improve the applications of nomograms. This study highlights the need for a renal size nomograms, specific for ethnic groups or geographical regions, which should be established with multi-centre studies, recruiting larger numbers of healthy adult subjects, representing different age groups, ethnicities, and geographical regions.

In conclusion, this preliminary study has provided normal renal lengths and kidney to body height ratios for a Sri Lankan young adult population, whilst underscoring the significance of developing renal nomograms for Sri Lankan population at larger. Further, considering the given gender and side differences in renal measurements, it is advisable to have gender as well as side specific renal nomograms.

This study should be considered as an initial step in developing such ultrasonic renal nomaograms for the Sri Lankan population.

\section{References}

1. Hammad LF. A sonographic study of kidney dimensions in Saudi's University Students. Pakistan J Med Sci, 2012; 28: 395-399.

2. Hekmatnia A, Yaraghi M. Sonographic measurement of absolute and relative renal length in healthy Isfahani adults. JRes Med Sci, 2004; 2: 1-4.

3. Miletić D, Fučkar Ž, Šustić A, et al. Sonographic measurement of absolute and relative renal length in adults. J. Clin. Ultrasound, 1998; 26: 185-189.

4. Balta JY, Cronin M, Cryan JF, et al. The utility of cadaverbased approaches for the teaching of human anatomy: A survey of British and Irish anatomy teachers. Anat Sci Educ, 2017; 10: 137-143.

5. Fraser KW. Effect of storage in formalin on organ weights of rabbits. N. Z. J. Zool, 1985; 12: 169-174.

6. Emamian SA, Nielsen MB, Pedersen JF. Intra observer and inter observer variations in sonographic measurements of kidney size in adult volunteers. A comparison of linear measurements and volumetric estimates. Acta Radiol, 1995; 36: 399-401.

7. Arooj A, Lam J, Wui YJ, et al. Comparison of renal size among different ethnicities. Int J Biol Biomed Eng, 2011; 5: 221-229.

8. Muthusami P, Ananthakrishnan R, Santosh P. Need for a nomogram of renal sizes in the Indian population-findings from a single centre sonographic study. IJMR, 2014; 139: 686-693.

9. Nadeeshani S, Dassanayake R, Kodithuwakku U. Ultrasonic Assessment of Kidney Length in a Sri Lankan Farming Population. Anuradhapura Med J, 2015; 9 (Supp 2): S07. Abstract.

10. Gunathilake SK, Samaratunga SS, Rubasinghe RT. Chronic kidney disease (CKD) in Sri Lanka - current research evidence justification: A review. Sabaragamuwa Univ J, 2015: 13: 31-33. 
11. El-Reshaid W, Abdul-Fattah H. Sonographic assessment of renal size in healthy adults. Med Princ Pract, 2014; 23: 432-436.

12. Oyuela-Carrasco J, Rodríguez-Castellanos F, Kimura E, et al. Renal length measured by ultrasound in adult mexican population. Nefrol publicacioǹ of la Soc Espanßla Nefrol 2009; 29: 30-34.

13. Buchholz NP, Abbas F, Biyabani SR, et al. Ultrasonographic renal size in individuals without known renal disease. J PakMedAssoc, 2000; 50: 12-16.

14. Okoye IJ, Agwu KK, Idigo FU. Normal sonographic renal length in adult southeast Nigerians. Afr J Med Med Sci, 2005; 34: 129-131.
15. Abdoelrahman HA, Mansour AA, Gar-elnabi MEM, et al. Ultrasonographic renal length and parenchymal thickness in normal Sudanese population. Int J Sci Res, 2016; 5: 623635.

16. Emamian SA, Nielsen MB, Pedersen JF, et al. Kidney dimensions at sonography: correlation with age, sex, and habitus in 665 adult volunteers. Am J Roentgenol, 1993; 160: $83-86$.

17. Ablett MJ, Coulthard A, Lee RE, et al. How reliable are ultrasound measurements of renal length in adults? $\mathrm{Br} \mathrm{J}$ Radiol, 1995; 68: 814-1087. 